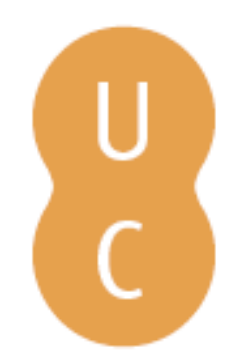

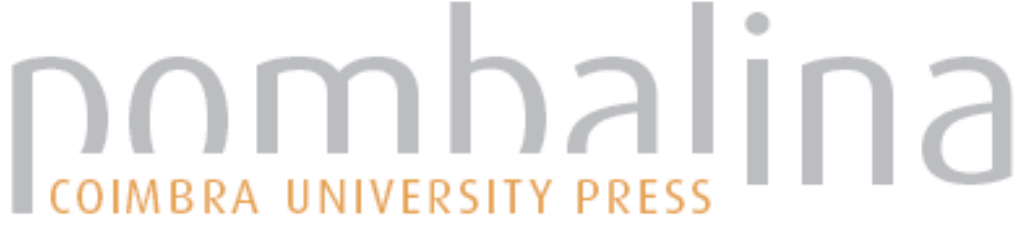

\section{Matemática da neurociência: de neurónios a circuitos e de circuitos a sistemas}

Autor(es): $\quad$ Gutkin, Boris; Pinto, David; Ermentrout, Bard

Publicado por: Imprensa da Universidade de Coimbra; Gravida

URL

persistente: URI:http://hdl.handle.net/10316.2/32615

DOI: $\quad$ DOI:http://dx.doi.org/10.14195/978-989-26-0485-5_11

Accessed : $\quad$ 26-Apr-2023 16:23:27

A navegação consulta e descarregamento dos títulos inseridos nas Bibliotecas Digitais UC Digitalis, UC Pombalina e UC Impactum, pressupõem a aceitação plena e sem reservas dos Termos e Condições de Uso destas Bibliotecas Digitais, disponíveis em https://digitalis.uc.pt/pt-pt/termos.

Conforme exposto nos referidos Termos e Condições de Uso, o descarregamento de títulos de acesso restrito requer uma licença válida de autorização devendo o utilizador aceder ao(s) documento(s) a partir de um endereço de IP da instituição detentora da supramencionada licença.

Ao utilizador é apenas permitido o descarregamento para uso pessoal, pelo que o emprego do(s) título(s) descarregado(s) para outro fim, designadamente comercial, carece de autorização do respetivo autor ou editor da obra.

Na medida em que todas as obras da UC Digitalis se encontram protegidas pelo Código do Direito de Autor e Direitos Conexos e demais legislação aplicável, toda a cópia, parcial ou total, deste documento, nos casos em que é legalmente admitida, deverá conter ou fazer-se acompanhar por este aviso. 
(Página deixada propositadamente em branco) 
MARIA PAULA SERRA DE OLIVEIRA

Coordenadora

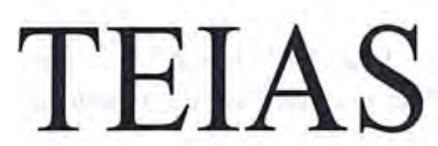

MATEMÁTICAS

Frentes na Ciência e na Sociedade 
(C) Gradiva - Publicações, L. da / Imprensa da Universidade de Coimbra, 2004 Coordenação editorial: Maria Paula Serra de Oliveira

Tradução: Artur Soares Alves

Carlota Isabel Leitão Pires Simões

Francisco José Craveiro de Carvalho

João Filipe Cortez Rodrigues Queiró

José Miguel Dordio Martinho de Almeida Urbano

Lia Sandra dos Santos

Mário da Silva Rosa

Paulo Eduardo Aragão Aleixo Neves de Oliveira

Revisão do texto: Isabel Pedrome

Capa: António Barros [Imprensa da Universidade. Coimbra], com imagem de

E. M. de Melo e Castro, "Fract 010 explod MC", Dezembro de 2003

[Fractal original gerado no Fractint com tratamento no Photoshop 7.0]

Infografia: Estúdios Estímulus [design]

Paginação: António Resende e Victor Hugo Fernandes

Impressão e acabamento: G.C. - Gráfica de Coimbra, L. da

Reservados os direitos para Portugal por:

Gradiva - Publicações, L. ${ }^{\text {da }}$ e Imprensa da Universidade de Coimbra

Gradiva - Publicações, L. ${ }^{d a}$

Rua Almeida e Sousa, 21, r/c, esq. • 1399-041 Lisboa

Telefs. $213974067 / 8 \cdot 213971357 \cdot 213953470$

Fax $213953471 \cdot$ Email: gradiva@ip.pt

URL: http://www.gradiva.pt

Imprensa da Universidade de Coimbra

Rua Antero de Quental, 195 • 3000-033 Coimbra

Telefs. 351239853110

Fax 3512398531 19 e-mail: fjrpress@ci.uc.pt

URL: http://www.imp.uc.pt

ISBN: 972-662-970-5

1." edição: Maio de 2004

Depósito legal n. ${ }^{\circ} 210431 / 04$

OBRA PUBLICADA COM O PATROCÍNIO DE:

CENTRO DE MATEMÁTICA DA UNIVERSIDADE DE COIMBRA DEPARTAMENTO DE MATEMÁTICA DA UNIVERSIDADE DE COIMBRA

FCT Fundação para a Ciência e a Tecnologia

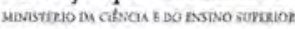


Boris Gutkin, David Pinto

Unité de Neurosciences Integratives et Computationelles, Institute Alfred Fessard, França

e Bard Ermentrout

Universidade de Pittsburgh, EUA

\section{Matemática da neurociência: de neurónios a circuitos e de circuitos a sistemas ${ }^{1}$}

\section{INTRODUÇÃ̃o}

Desde há muitos anos que a matemática e os métodos computacionais têm vindo a desempenhar um papel importante no nosso conhecimento do sistema nervoso. O objectivo deste capítulo consiste em apresentar alguns exemplos que mostram a forma como certas técnicas matemáticas podem ser aplicadas, a vários níveis, para aumentar o nosso conhecimento sobre os sistemas neuronais. Começaremos com uma descrição dos princípios biofísicos subjacentes à formação do potencial de acção em neurónios individuais. A ideia fundamental da modelação é representar as propriedades eléctricas de membranas biológicas através de um circuito equivalente formado por condensadores e resistências ligados em paralelo. Utilizaremos depois métodos do domínio das equações diferenciais para estudar um modelo simplificado de um neurónio individual e mostrar como se pode reduzir a dinâmica no plano a um sistema dinâmico escalar

${ }^{1}$ Tradução de Mário da Silva Rosa, professor do Departamento de Matemática, Universidade de Coimbra. Revisão de F. Geraldes, professor do Departamento de Bioquímica, Universidade de Coimbra, e E. Quinta Ferreira, professora do Departamento de Física, Universidade de Coimbra. 
num círculo. Utilizam-se simulações do modelo reduzido para explicar a estatística da formação de pontas (spikes) de neurónios individuais accionados por estímulos ruidosos. Seguidamente, analisaremos circuitos neuronais simples, envolvendo redes de neurónios excitatórios e inibitórios. Uma aproximação de campo médio reduz estas redes a um sistema planar e a análise do plano de fases permite-nos explicar resultados experimentais do sistema somato-sensorial (tacto) do rato. Finalmente, examinaremos redes alargadas, organizadas espacialmente. Aplicaremos a teoria da bifurcação a estas redes e, com base nos resultados, explicaremos os padrões observados durante alucinações visuais.

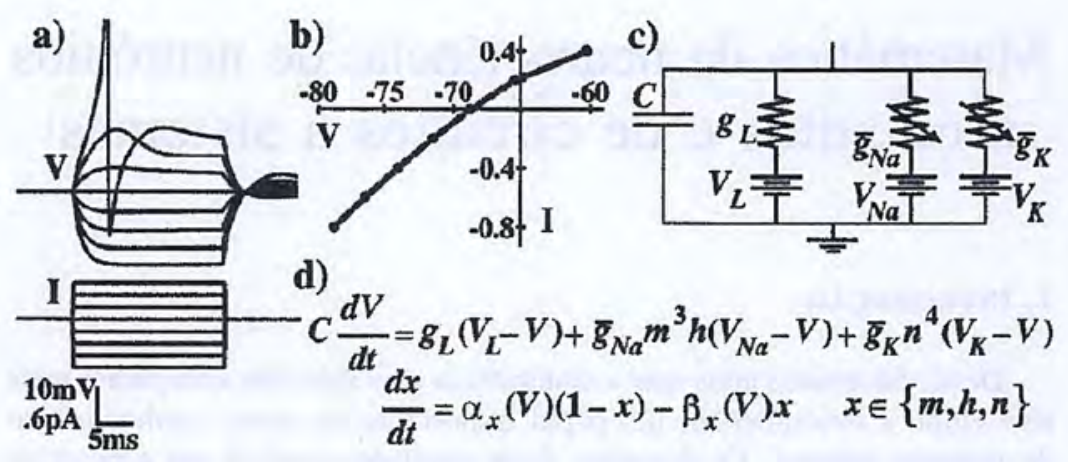

Fig. 1-Modelo de circuito equivalente de actividade neuronal a) Alterações do potencial de membrana neuronal $(V)$ em resposta a diferentes niveis de corrente injectada (I). b) Relação I-V de respostas neuronais. c) Diagrama do circuito que representa correntes capacitivas e resistivas, activas e passivas. d) Equações de Hodgkin Huxley do equilibrio de corrente e da cinética dos canais com «portas» (gating kinetics)

\section{Modelos do Circuito equivalente}

Para representar a dinâmica da actividade eléctrica observada em neurónios individuais tornou-se corrente a utilização do modelo de circuito equivalente. Este modelo é baseado na ideia de que a actividade neuronal pode ser completamente descrita pelo fluxo de diferentes correntes associadas à membrana neuronal. As correntes dividem-se em dois tipos: aquelas que podem ser representadas por elementos lineares do circuito (correntes passivas) e as que são dependentes do potencial do tempo e 
requerem uma dinâmica mais complexa (correntes activas). Ambos os conjuntos podem ser entendidos em termos das observações experimentais apresentadas na figura 1a) (ver o trabalho de revisão de Johnston e Wu, 1997).

\subsection{Correntes passivas}

A primeira observação é que os neurónios mantêm uma diferença de potencial constante através das suas membranas, o que se designou por «potencial de repouso», $(\mathrm{V} m \approx-65 \mathrm{mV})$. Este potencial deve-se: 1) ao facto de as membranas neuronais serem semipermeáveis, permitindo que apenas certos iões passem de um lado para o outro (maioritariamente sódio, $\mathrm{Na}^{+}$, potássio, $\mathrm{K}^{+}$, e cálcio, $\mathrm{Ca}^{++}$); 2) ao facto de os neurónios manterem activamente um gradiente de concentração daqueles mesmos iões, através da membrana; 3 ) ao facto de os iões em causa possuírem uma carga eléctrica. Por exemplo, o potássio, $\mathrm{K}^{+}$, tem uma concentração maior no interior da célula e uma carga positiva. Forças de difusão tendem a fazer sair $\mathrm{K}^{+}$da célula. A perda subsequente de iões positivos conduz ao aparecimento de uma carga negativa no interior da membrana. A força eléctrica resultante atrai iões positivos, incluindo os ligados a $\mathrm{K}^{+}$, novamente para dentro da célula. O potencial de repouso, também designado por potencial de equilíbrio, é o nível de potencial para o qual as forças eléctricas e de difusão, devidas a todos os iões permeáveis, estão em equilíbrio. $\mathrm{O}$ elemento de circuito equivalente, utilizado para representar este potencial, é uma força electromotriz (FEM) ou bateria.

A segunda observação é que a injecção directa de corrente num neurónio induz uma variação incremental no potencial. Para uma certa gama de intensidades de corrente, a variação induzida no potencial é linear (figura 1b), o que, de acordo com a lei de Ohm (V = IR), sugere que a membrana está, em parte, a funcionar como uma resistência linear (as não linearidades na relação serão analisadas posteriormente). Experimentalmente, a conductância da membrana é medida a partir do declive da linha obtida através da representação gráfica da variação do potencial induzida por intensidades diferentes de corrente injectada. Ainda que muitos iões diferentes contribuam para a alteração do potencial, utiliza-se frequentemente uma única conductância de fuga (leak conductance), $g_{L}$, para contemplar todas as correntes iónicas passivas.

A terceira observação é que as variações do potencial, devido à injecção de corrente, se comportam exponencialmente no tempo. Isto sugere que as membranas neuronais operam como um circuito-RC, isto é, uma resistência linear em paralelo com um condensador. 
As membranas neuronais são compostas por uma dupla camada de moléculas lipídicas na qual estão imersas proteínas. As proteínas possuem resistividades específicas baixas $(\sim 1 \Omega \mathrm{cm})$ e formam os canais proteicos através dos quais o fluxo de iões carregados atravessa a membrana. Por outro lado, os lípidos têm resistividades específicas elevadas $\left(\sim 10^{9} \Omega \mathrm{cm}\right)$, formando um isolamento eficiente entre as soluções celulares interna e externa, altamente electrolíticas, o que constitui exactamente o arranjo característico de um condensador de placas paralelas. A corrente que é injectada na membrana divide-se numa corrente capacitiva que carrega a bicamada lipídica e numa corrente iónica que passa através dos canais proteicos. Para um circuito- $\mathrm{RC}$, a constante tempo da variação do potencial é $\tau=\mathrm{RC}$. Portanto, experimentalmente, a capacidade de um neurónio é obtida como $\mathrm{C}=\tau / \mathrm{R}$, onde $\tau$ é a constante de tempo observada correspondente à taxa de variação exponencial do potencial de membrana e $\mathrm{R}$ é a resistência medida do modo acima descrito.

\subsection{Correntes activas}

A maior parte das correntes observadas exibem uma condutância constante apenas numa certa gama de potenciais. De facto, é de esperar alguma não linearidade na relação $\mathrm{I}-\mathrm{V}$, mesmo no caso de correntes totalmente passivas. Por exemplo, quando o potencial é tal que ambas as forças, de difusão e eléctrica, produzem movimentos de iões na mesma direcção, a condutância observada pode ser maior do que aquela que uma simples relação linear faria prever. Em muitos casos, contudo, a não linearidade, ou rectificação, numa condutância de canal não pode ser explicada apenas por propriedades passivas. Além disso, observa-se frequentemente que a conductância depende não só do potencial como também do tempo (ver figura 1a).

A fim de explicar a rectificação anómala observada em muitas correntes neuronais, Hodgkin e Huxley (1952) propuseram o modelo de condutância do canal com portas (gating model). A ideia é que alguns canais de proteínas, que permitem a passagem de iões através da membrana, possuem também partículas carregadas que abrem ou fecham o canal (gating particles) de uma forma dependente do potencial e do tempo, alterando assim a condutância. Por exemplo, a condutância de correntes de $\mathrm{K}^{+}$activas é representada por uma condutância máxima $g_{K}$ vezes $n^{4}$, onde $n$ é a probabilidade de que uma partícula-porta (gating particle) esteja na posição que contribui para a abertura do canal e o expoente 4 indica o número destas partículas por canal. 
A dinâmica das partículas-porta (gating particles) é modelada usando cinéticas de primeira ordem, como se mostra na figura 1d, onde $\alpha(V)$ é a taxa, dependente do potencial, à qual os canais abertos se fecham e $\beta(V)$ é a velocidade à qual os canais fechados se abrem. No caso de correntes de $\mathrm{Na}^{+}$activas, as experiências sugerem que há dois tipos de partículas-porta independentes envolvidas, três de activação, $m$, e uma de inactivação, $h$. A dependência do potencial e do tempo de cada «porta» podem determinar-se experimentalmente examinando as alterações de condutância associadas a cada corrente individual. O elemento de circuito equivalente utilizado para representar a condutância neuronal activa é uma resistência variável.

\subsection{Equações de equilíbrio de corrente}

Consideradas conjuntamente, as correntes passiva e activa acima descritas caracterizam o circuito equivalente ou modelo de condutância em paralelo de actividade neuronal, mostrado na figura 1c. A equação que descreve a dinâmica do circuito é uma consequência da primeira lei de Kirchoff: é nula a soma de todas as correntes que convergem num nodo. Em particular, uma corrente aplicada divide-se numa corrente capacitiva que carrega a membrana e em correntes resistivas que passam através dos canais activos e passivos. Normalmente, a equação é escrita como se indica na figura $1 \mathrm{~d}$, em que a corrente capacitiva $(C . d V / d t)$ é igual à soma das várias correntes iónicas.

O grande mérito do modelo de Hodgkin e Huxley foi a sua capacidade de explicar muito mais do que a sensibilidade das várias condutâncias neuronais ao potencial e ao tempo. Uma vez correctamente caracterizada cada uma das correntes, constatou-se que os mesmos mecanismos não lineares das «portas» eram suficientes para explicar a formação de potenciais de acção, isto é, pontas (spikes) de potencial «tudo ou nada» explosivos que dão início à sinalização sináptica entre neurónios. Utilizando o mesmo conjunto de equações de equilíbrio de corrente, Hodgkin e Huxley demonstraram como os potenciais de acção resultam duma sequência estereotipada de variações de condutâncias entre as várias correntes iónicas.

Além de $\mathrm{Na}^{+}$e $\mathrm{K}^{+}$, foram também descritas outras condutâncias que dependem do potencial e do tempo. Considera-se que os canais de $\mathrm{Ca}^{++}$ estão na base da formação de trens de potenciais de acção que caracterizam a actividade nalguns neurónios do tálamo e do córtex cerebral (McCormick e Huguenard, 1992). O decréscimo no tempo da taxa de 
formação de potenciais de acção (spike adaptation) depende de canais de $\mathrm{K}^{+}$que são sensíveis não apenas ao potencial, mas também à concentração de $\mathrm{Ca}^{++}$no interior do neurónio (Lancaster e Adams, 1986). Os canais de $\mathrm{Na}^{+}$que funcionam numa escala de tempo mais lenta e não têm porta de inactivação podem ser responsáveis pelo aumento de sinais que chegam de outros neurónios (Stafstrom et al., 1982). Cada uma destas correntes pode ser incorporada no modelo de equilíbrio de corrente, com a adição de condutâncias e cinéticas apropriadas, ao conjunto de equações.

\subsection{Controlo dinâmico (Dynamic Clamp)}

Uma aplicação recente e muito interessante das equações de equilibrio de corrente está relacionada com a modificação da função de neurónios reais. Isto é feito com modelos computacionais em tempo real e o método designa-se por «dynamic clamp» ou «modelo híbrido» (Sharp et al., 1993). A ideia subjacente é ligar neurónios reais a correntes iónicas simuladas, ou mesmo a membranas completamente simuladas. Utiliza-se um eléctrodo para medir o potencial de membrana de um neurónio real. Esta medida é usada para calcular correntes iónicas simuladas. Estas são depois novamente injectadas no neurónio através do mesmo eléctrodo. As aplicações mais comuns da técnica consistem em «inserir» novos canais eléctricos na membrana de um neurónio ou em «subtrair» um canal existente e estudar o respectivo efeito no comportamento do neurónio. Inicialmente o método foi aplicado ao estudo dos neurónios no sistema digestivo da lagosta (Sharp et al. 1993), enquanto em estudos mais recentes se tem examinado o comportamento de neurónios individuais no cérebro de mamíferos (Jaeger e Brower, 1999). Uma outra aplicação que está actualmente a ser desenvolvida consiste em utilizar o método do modelo híbrido na construção de circuitos de neurónios simulados, modelados em «microchips» analógicos programáveis (Le Masson et al., 1995). Colaborações recentes têm explorado esta técnica para estudar como controlam os circuitos neuronais os ritmos cerebrais durante o sono (Le Masson, comunicação pessoal).

\section{Planos de fases e «SPIKING»}

O modelo de equilíbrio de corrente de Hodgkin e Huxley acima descrito é um sistema dinâmico a quatro dimensões, o que torna difícil a sua análise matemática rigorosa. Morris e Lecar (ver Rinzel e Ermentrout, 
1998) construíram um modelo de neurónio muito simples baseado apenas em três condutâncias: um canal de cálcio rápido, um canal de potássio lento e um canal de fuga passivo. Com base na formulação do circuito equivalente acima descrita, as equações a considerar são:

$$
\begin{aligned}
\mathrm{C} \frac{d V}{d t} & =\mathrm{I}+\mathrm{g}_{\mathrm{L}}\left(\mathrm{V}_{\mathrm{L}}-\mathrm{V}\right)+\mathrm{g}_{\mathrm{Ca}} \mathrm{m}_{\infty}(\mathrm{V})\left(\mathrm{V}_{\mathrm{Ca}}-\mathrm{V}\right)+\mathrm{g}_{\mathrm{K}} \omega\left(\mathrm{V}_{\mathrm{K}}-\mathrm{V}\right) \\
\frac{d \omega}{d t} & =\frac{\omega_{\infty}(V)-\omega}{\tau_{\omega}(V)} .
\end{aligned}
$$

Sendo suficientemente rápidas, pode considerar-se que as cinéticas das «portas» do canal de cálcio, $m_{\infty}(\mathrm{V})$, actuam instantaneamente. Assim, o sistema requer apenas duas variáveis dinâmicas e pode ser analisado, de uma forma rigorosa e completa, através de métodos associados ao plano de fases.

Estes métodos são dos mais úteis que surgiram na neurociência teórica oriundos da teoria qualitativa de equações diferenciais. O objectivo é compreender a dinâmica de duas variáveis, representando graficamente uma como uma função da outra. A designação de plano de fases deriva do facto de, através da representação gráfica de duas variáveis dinâmicas, em particular das que têm alguma espécie de comportamento periódico ou estereotipado, podermos estudar a «fase» relativa de uma das variáveis face à outra. Por exemplo, consideremos um neurónio formando um potencial de acção. O potencial de membrana afasta-se de um nível de repouso até atingir o nível máximo do potencial, voltando a descer através de um processo de queda ou pós-hiperpolarização, regressando então à posição de repouso. A variável de recuperação, $\omega$, segue uma trajectória correspondente em resposta ao potencial de membrana. Se representarmos num gráfico o potencial face à variável de recuperação $\omega$ (ver figura $2 \mathrm{a}$ e b), o resultado é uma curva fechada, ou trajectória, no plano de fases.

O plano de fases é caracterizado por várias marcas ou pontos especiais que ajudam a definir e a visualizar as dinâmicas. Por exemplo, as linhas de inclinação nula do sistema são definidas como as curvas ao longo das quais uma ou a outra variável permanece constante. A curva ao longo da qual $d V / d t=0$, define a linha de inclinação nula $V$, enquanto $d \omega / d t=0$ define a linha de inclinação nula $\omega$. Nos pontos onde aquelas duas linhas se intersectam, nenhuma das variáveis se altera; os pontos de intersecção definem os estados de equilíbrio do sistema. Um tal ponto, marcado por $\mathrm{R}$, corresponde ao potencial de repouso do neurónio. Trata-se de um ponto fixo atractivo: pequenas perturbações transitórias no potencial de 
membrana, por intermédio, por exemplo, da aplicação de uma corrente externa ou por activação de conductâncias que dependem do potencial, serão amortecidas com o tempo e o potencial voltará ao ponto de repouso. Um outro ponto do estado de equilíbrio é o assinalado com $\mathrm{T}$ - potencial de limiar do neurónio. Este é um ponto fixo repulsivo, pelo que as trajectórias que se aproximem de $\mathrm{T}$ retornam à posição de repouso ou continuam, após terem produzido um potencial de acção.
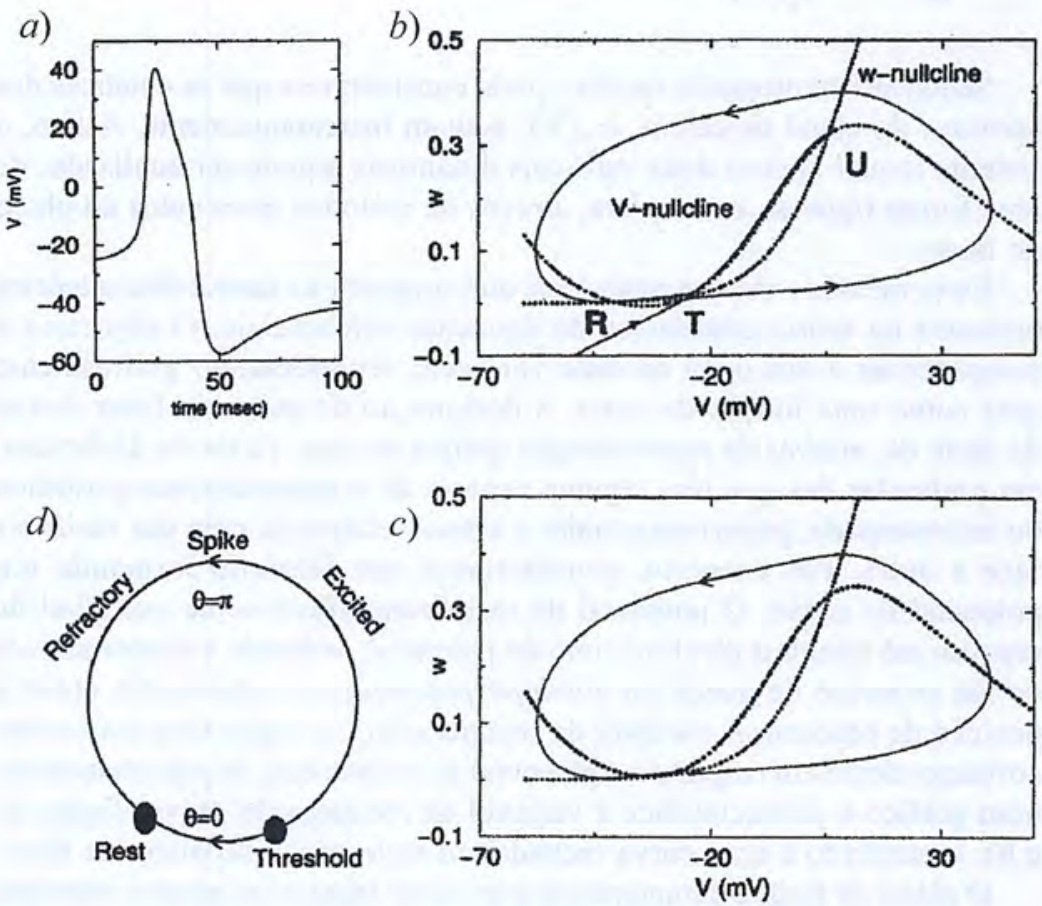

Fig. 2 - a) Um potencial de acção, ou ponta, no potencial de membrana representado graficamente no tempo; b) Diagrama de fases do mesmo potencial de acção. Nele estão representadas as linhas de inclinação nula $V$ e $\omega$. As suas intersecções correspondem a pontos fixos: $R$ é o ponto atractivo, Té o limiar que corresponde a um ponto-sela (atractivo numa direcção e repulsivo noutras direcções), U é um ponto repulsivo; c) Após o aumento da corrente injectada, a linha de inclinação nula $V$ cresce e não existem pontos de repouso estáveis, mas sim um ciclo-limite atractivo correspondente ao estado de explosão; d) Diagramas de estado equivalentes para o neurónio-teta. A cheio marcam-se os pontos críticos do modelo - R e T. O modelo inclui também o potencial de acção e o comportamento refractário do modelo completo 
O plano de fases permite visualizar o efeito da injecção de correntes num neurónio. Matematicamente, injectar uma corrente estável positiva corresponde a adicionar uma constante positiva ao segundo membro da equação do potencial. No plano de fases, o potencial da linha de inclinação nula $V$ é aumentada, o que altera a posição dos pontos fixos. Quando o potencial daquela linha cresce suficientemente, os estados estacionários $\mathrm{R}$ e T convergem e depois desaparecem. Isto revela uma solução periódica ou solução de ciclo-limite no plano de fases correspondente à formação repetitiva de potenciais de acção, ou disparo, pelo neurónio. À semelhança dos estados de equilíbrio estável, os ciclos-limite podem ser atractivos ou repulsivos. Apenas as oscilações estáveis podem ser realizadas fisiologicamente (excepto quando se aplicam controlos especiais), mas as soluções periódicas instáveis podem actuar como separatrizes, dividindo o plano de fases em bases de atracção para múltiplos atractores. Neste modelo particular, notemos que as linhas de inclinação nula podem estar arbitrariamente próximas uma da outra (figura $2 \mathrm{c}$ ), pelo que a frequência das oscilações pode ser arbitrariamente baixa. $\mathrm{O}$ aumento de corrente aplicada conduz a frequências mais elevadas na medida em que o «canal» entre as linhas de inclinação nula se alarga.

Um pormenor importante e um pouco surpreendente é que a trajectória do ciclo-limite permanece constante na sua forma, independentemente de quão rápidas são as explosões da célula (ou atravessa a curva fechada). Do ponto de vista experimental, isto corresponde à forma invariante dos potenciais de acção gerados. Em termos matemáticos, permite-nos considerar aquela linha fechada como uma estrutura invariante que não se altera com a dinâmica dos sistemas. Por este motivo, se conseguirmos um processo para marcar apenas as quantidades que se alteram, deveremos ser capazes de simplificar o modelo.

Formalmente, isto é feito através do método de formas normais da teoria da bifurcação. Descreveremos apenas as ideias heurísticas que lhe estão subjacentes.

Como os dois pontos fixos $\mathrm{R}$ e $\mathrm{T}$ convergem e desaparecem e o ciclo-limite emerge, o sistema aproxima-se de um ponto de bifurcação nas soluções dinâmicas. Quando examinamos os valores próprios do modelo linearizado próximo desta bifurcação constatamos que um único valor próprio real vai para 0 . Isto significa que a bifurcação é a de um nodo-sela num círculo invariante («saddle-node»), o que fornece a base matemática para tratar a trajectória circular como um invariante. Para encontrar o «centro» matemático desse círculo, descrevemos a variação do potencial à sua volta, utilizando uma variável angular $\theta$. Consideramos este ângulo igual a zero $(\theta=0)$ quando o nível do potencial é o de 
repouso, no ponto $\mathrm{R}$, e consideramos que $\theta$ cresce à volta do círculo na direcção contrária à do movimento dos ponteiros do relógio. $\mathrm{O}$ potencial de acção é então uma revolução do ângulo relativo $\omega / \mathrm{v}$ ou «fase» de 0 até $\pi$, voltando a $2 \pi$. O artifício está em encontrar uma transformação matemática das variáveis do modelo original do circuito equivalente, tal que uma das novas variáveis percorra o ciclo-limite invariante e as restantes variáveis sejam ortogonais àquela. Para um sistema dinâmico com uma bifurcação nodo-sela, uma tal transformação pode sempre ser encontrada e conduz à equação:

$$
\frac{d x}{d t}=q x^{2}+p I
$$

onde a variável $x$ descreve a dinâmica, $p$ e $I$ são parâmetros que incluem, por exemplo, a influência de «inputs» externos, e $q>0$ é um parâmetro que depende dos detalhes do modelo original completo. Esta equação é um tanto incómoda, uma vez que a sua solução se torna infinita em tempo finito. Tratamos a singularidade envolvendo $x$ num círculo utilizando a mudança de variáveis $x=\tan \theta / 2$, chegando assim matematicamente à variável de fases acima descrita. A equação final para o modelo em $\theta$ vem:

$$
\frac{d \theta}{d t}=q(1-\cos \theta)+(1+\cos \theta) p I .
$$

Este modelo é muito simples; contudo, reproduz o comportamento do modelo mais complexo do circuito equivalente. De facto, o neurónio-teta é um modelo canónico para uma vasta classe de modelos baseados em condutâncias que exibem dinâmicas de bifurcação com nodo-sela. Mais especificamente, qualquer modelo de um neurónio baseado em condutâncias que exiba uma bifurcação nodo-sela pode ser descrito pelo neurónio-teta. Por isso, se conseguirmos compreender o comportamento do neurónio-teta, saberemos mais acerca da função de muitos tipos diferentes de neurónios. Por exemplo, eis algumas das propriedades do neurónio-teta que reflectem o comportamento geral de neurónios piramidais encontrados no neocortex:

- Os potenciais de acção são acontecimentos do tipo tudo ou nada, ou seja, quando estimulada, a actividade abaixo do limiar T regressa ao repouso. Mas, uma vez passado o ponto $\mathrm{T}$, ocorre uma ponta (spike) completa.

- O modelo e os neurónios geram sequências contínuas de potenciais de acção em resposta a injecções de correntes constantes.

- Surgem explosões repetitivas com frequências arbitrariamente baixas. 
- A curva (IF) da relação corrente de entrada/frequência de saída pode ser facilmente ajustada por uma raiz quadrada (IF instantânea) ou por uma função linear (IF estacionária), ambas observadas em neurónios reais.

Todas estas propriedades são básicas e nos mostram que o modelo reduzido contém «informação» dinâmica sobre os neurónios reais. A seguir utiliza-se este modelo reduzido para explorar a dinâmica subjacente a comportamentos mais complexos.

\section{EstatístiCa de aCTIVIDADE NEURONAL DO CÓRTEX «IN VIVO»}

Um debate de longa data em neurociềncia prende-se com a questão de saber se os neurónios codificam a informação acerca do mundo em termos da frequência média de formação de potenciais de acção ou através da distribuição precisa no tempo de potenciais de acção individuais. A segunda hipótese tem sido considerada improvável, uma vez que o disparo de neurónios no cérebro vivo é muito irregular (ver, por exemplo, Shadlen e Newsome, 1998). Por outro lado, várias experiências mostraram que os neurónios são capazes de produzir de um modo fidedigno potenciais de acção definidos no tempo. Numa experiência, em particular, foi demonstrado que injectando niveis constantes de corrente os neurónios formam potenciais de acção a uma velocidade contida num certo conjunto de valores, mas com distribuição temporal irregular. Contudo, após repetidas injecções de corrente com ruído, os tempos particulares de disparo foram muito mais precisos relativamente ao sinal de entrada (Mainen e Sejnowski, 1995). Os resultados do modelo demonstram que o neurónio-teta apresenta respostas semelhantes para sinais de entrada constantes versus sinais de entrada com ruídos (figura 3) (Gutkin e Ermentrout, 1998). Examinaremos aqui o modo como o modelo permite captar e explicar estas conclusões não intuitivas.

O neurónio-teta com potencial de repouso com ruído mas com corrente de entrada constante é basicamente um oscilador intrínseco perturbado por um efeito de potencial aleatório ou, em linguagem matemática, por um processo de renovação não-linear. Assim, o tempo de partida de cada ciclo sucessivo de potenciais de acção depende do tempo do potencial de acção anterior. Uma vez que estes tempos são perturbados pela variação do «potencial», a incerteza aumenta em cada potencial de acção sucessivo. De facto, pode mostrar-se em termos analiticos (Gutkin e Ermentrout, 1998) e numéricos (figura 3) que esta incerteza, medida como a variância 
de tempos de potenciais de acção em cada experiência, é representada pela raiz quadrada do número de potenciais de acção multiplicado pela variância do tempo do primeiro potencial de acção.

a)
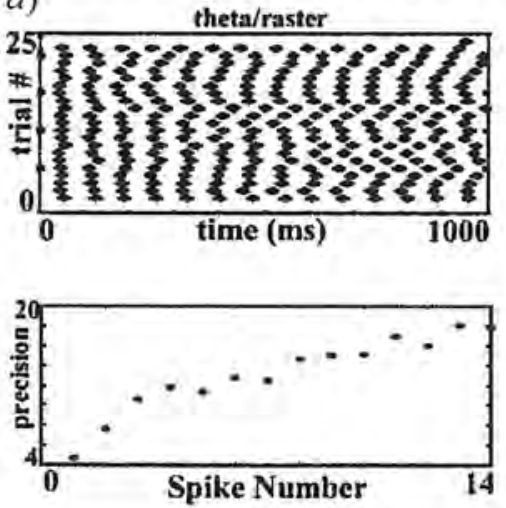

b)
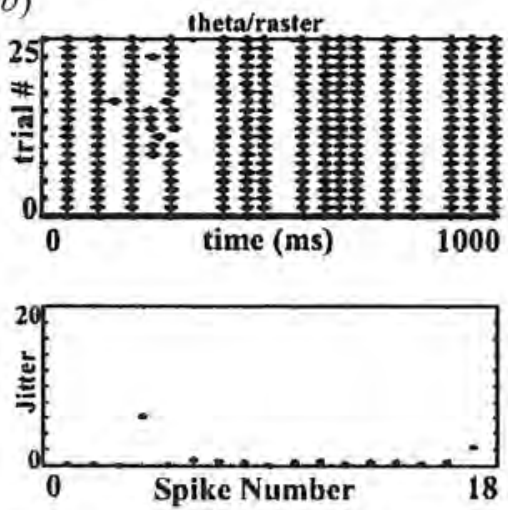

Fig. 3 - a) Resposta do modelo de neurónio-teta (com potencial de repouso com ruido) quando estimulado por uma corrente constante. O gráfico mostra os tempos de formação de potenciais de acção para ensaios repetidos com o mesmo estimulo consiante. Os primeiros potenciais de acção aparecem com a mesma distribuiçâo enquanto os ültimos se deslocam aleatoriamente. Em baixo mostra-se o gráfico da incerteza, nos tempos dos potenciais de acção, calculados como o desvio padrâo dos tempos obtidos nos ensaios. Como se pode constatar, a incerteza aumenta em cada potencial de acção sucessivo.

b) Resposia do mesmo modelo de neurónio-teta quando estimulado repetidamente por uma corrente perlurbada (variada). Os potenciais de acção permanecem consistentes durante cada ensaio (gráfico superior) e a incerteza mantém-se baixa de potencial de acção para potencial de acção (gráfico inferior)

Por outro lado, quando o mesmo neurónio-teta é estimulado por correntes repetidas com ruído, os tempos dos potenciais de acção não dependem uns dos outros, mas da dinâmica dos estímulos. Se o estimulo for de tal forma que conduza rapidamente o potencial para além de $\mathrm{T}$, então forma-se um potencial de acção para o mesmo ponto de entrada em todos os ensaios. Desta forma, a incerteza é baixa e não aumenta em cada potencial de acção.

Isto significa que, para que os neurónios codifiquem informação com o tempo de formação do potencial de acção preciso, deverão fazê-lo em resposta a entradas de corrente do tipo choque. Ao mesmo tempo, entradas lentas serão codificadas a uma velocidade de disparo neuronal média. 
A análise em curso do neurónio-teta sugere que estes dōis esquemas de codificação poderão não ser mutuamente exclusivos, mas que ambos os modos podem contribuir para a saída de potenciais de acção de um neurónio individual (Gutkin e Ermentrout, em preparação).

\section{Modelos de ACTIVIDAdE}

As secções anteriores mostraram como reduzir as equações de equilíbrio de corrente a um simples modelo escalar para compreender a dinẩmica de neurónios individuais. Em muitos casos, relevantes a nível experimental, é desejável ir mais além na simplificação da dinâmica, utilizando os chamados modelos de actividade ou modelos de velocidade de disparo. Nestes modelos, a quantidade relevante não é o tempo de formação do potencial de acção ou o potencial de um neurónio individual, mas antes o nivel de actividade generalizada ou velocídade de disparo em neurónios individuais ou em populações neuronais.

Em contraste com a representação biofísica fornecida pelas equações de equilíbrio de corrente, os modelos de actividade baseiam-se numa descrição mais funcional da actividade neuronal. Estes modelos são sobretudo utilizados para estudar interacções entre vastas populações neuronais, ou, mais geralmente, nos casos em que um modelo biofísico se torna impraticável ou muito complexo. Nesta secção apresentaremos uma derivação funcional do modelo de actividade e descreveremos um exemplo em que é habitualmente utilizado para examinar o processamento de sinais de entrada sensoriais nos bigodes dos roedores.

\subsection{Dedução das equaçōes}

A equação central dos modelos de actividade descreve a relação entre três medidas distintas de actividade neuronal: potencial, velocidade de disparo e impulso sináptico (descrito em baixo). A formação de um potencial de acção ou o disparo de um neurónio activa ligações sinápticas transmitidas a outros neurónios. As sinapses activadas induzem alterações de potencial ou potenciais pós-sinápticos (PPS) nos neurónios receptores. Os PPS são muitas vezes representados como seguindo um curso temporal característico, descrito através de uma função «alfa», $\alpha(t)$ (Rall, 1998). Os PPS podem ser positivos ou negativos, dependendo de a sinapse ser excitatória (EPPS) ou inibitória (IPPS). Para uma série de potenciais de 
acção, as funções alfa somam-se e o potencial, V, no neurónio pós-sináptico é dado por

$$
V(t)=\sum_{i} \alpha\left(t-t_{i}\right)
$$

em que $i$ corresponde ao número de potenciais de acção e $t_{i}$ é o tempo de chegada para cada um deles. Numa população de neurónios, a distribuição de potenciais de acção no tempo é descrita frequentemente através de uma função contínua que representa a velocidade média de disparo dessa população, $F(t)$. Esta função depende do nível médio do potencial, pelo que $F(t)=F(V(t))$. Se separar a população em neurónios excitatórios versus neurónios inibitórios, a equação para o potencial excitatório médio é dada por

$$
V_{e}(t)=e e \int_{-\infty}^{t} \alpha_{e}\left(t-t^{\prime}\right) F_{e}\left(V_{e}\left(t^{\prime}\right)\right) d t^{\prime}-i e \int_{-\infty}^{t} \alpha_{i}\left(t-t^{\prime}\right) F_{i}\left(V_{i}\left(t^{\prime}\right)\right) d t^{\prime} .
$$

Nesta equação as duas populações têm distintas funções $\alpha, F$ de taxas de disparo e ie (por exemplo) representa a eficácia relativa de sinapses da população inibitória para a excitatatória. O potencial médio da população inibitória é definido de modo semelhante.

Pode exprimir-se uma formulação alternativa do modelo de actividade, se definirmos o impulso sináptico de cada população por

$$
\begin{gathered}
S_{e}=\int_{-\infty}^{t} \alpha_{e}\left(t-t^{\prime}\right) F_{e}\left(V_{e}\left(t^{\prime}\right)\right) d t^{\prime} \\
S_{i}=\int_{-\infty}^{t} \alpha_{i}\left(t-t^{\prime}\right) F_{i}\left(V_{i}\left(t^{\prime}\right)\right) d t^{\prime}
\end{gathered}
$$

pelo que, tomando novamente a população excitatória como exemplo,

$$
S_{e}=\int_{-\infty}^{t} \alpha_{e}\left(t-t^{\prime}\right) F_{e}\left(e e S_{e}-i e S_{i}\right) d t^{\prime}
$$

Além disso, se admitirmos que as funções alfa são descritas por simples funções exponenciais decrescentes (e.g. $\alpha_{e}=e^{-t / \tau_{e}}$ ), então podemos diferenciar para obter,

$$
\tau_{e} \frac{d S_{e}}{d t}+S_{e}=F_{e}\left(e e S_{e}-i e S_{i}\right)
$$

A equação (3) é designada formulação do potencial e a equação (4) é a formulação da actividade (Ermentrout, 1997). Intuitivamente, o termo impulso sináptico deriva das unidades de $e e S_{e}$, descrevendo a alteração de potencial induzida por sinapses excitatórias. Na maior parte dos modelos, ee mede-se em unidades de volts $\times$ sinapse, a eficácia da ligação 
vezes o número de sinapses. Assim, $\mathrm{S}_{\mathrm{e}}$ mede-se na unidade $1 /$ sinapse, uma quantidade adimensional por sinapse, i.e., impulso sináptico (Pinto et al., 1996). Como se descreve a seguir, a formulação da actividade permite, em particular, a incorporação directa de dados biológicos no sistema modelo.

\subsection{Bigodes e barris}

Ao deflectir-se um bigode na face de um roedor, activa-se uma cadeia de neurónios desde a periferia, passando pelo eixo do cérebro, o tálamo e seguindo até um feixe de neurónios definido anatomicamente no neocortex, normalmente designado por «barril de bigodes» (Woolsey e van der Loos, 1970). Cada barril contém duas populações principais de células, neurónios excitatórios e neurónios inibitórios. Ambas as populações recebem do tálamo sinais sinápticos excitatórios. Além disso, ambas as populações estabelecem ligações recíprocas com neurónios do outro tipo e contactos recorrentes a neurónios do mesmo tipo (figura 4a) (Simons, 1978). Os neurónios excitatórios no barril também formam contactos sinápticos com neurónios situados noutras regiões do cérebro (White, 1989). Portanto, utilizando quer o modelo de actividade quer os dados experimentais, o objectivo é compreender como é que interacções as sinápticas no interior de um barril operam nos sinais de entrada provenientes do tálamo e os transformam em sinais de saída que emanam da população de neurónios excitatórios.

a)

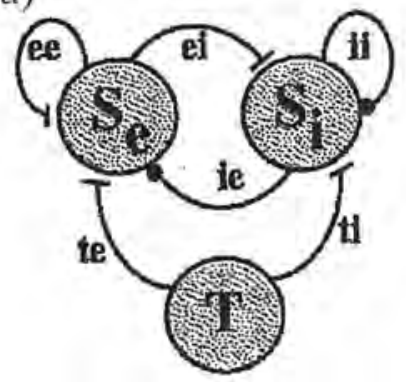

b)

$$
\begin{gathered}
\tau_{e} \frac{d S_{e}}{d t}+S_{e}=F_{e}\left(e e S_{e}-i e S_{i}+t e T\right) \\
\tau_{i} \frac{d S_{i}}{d t}+S_{i}=F_{i}\left(e i S_{e}-i i S_{i}+i i T\right) \\
\tau_{e} \frac{d T}{d t}+T=\text { THAL }(t) .
\end{gathered}
$$

Fig. 4-Modelo de actividade do barril-cortex de um roedor- a) Diagrama esquemático de ligaçòes sinápticas entre populaçôes excitatórias $\left(S_{e}\right)$ e inibitórías $\left(S_{i}\right)$ do barril e do tálamo (T). As sinapses excitatórias são representadas por linhas e as sinapses inibitórius por pontos: b) Formulaçào da actividade do bartil incorporando a entrada do tálamo 
Experiências laboratoriais medem o número e o tempo de potenciais de acção gerados por neurónios do tálamo e do barril em resposta a uma deflecção. Geralmente, reúnem-se os dados de muitos neurónios e calcula-se a velocidade média de disparo para cada população. A utilidade do modelo de actividade deriva do facto de representar a actividade neuronal de uma forma compatível com estes dados experimentais. Uma vez que tanto os dados das populações de neurónios do tálamo como dos neurónios do barril são medidos em termos de velocidade média de disparo, os dados talâmicos podem ser utilizados directamente como sinais de entrada para o modelo (THAL(t)), tal como os dados da população de neurónios excitatórios do barril o podem ser para avaliar a saída excitatória do modelo ( $\mathrm{F}_{\mathrm{e}}$, figura $4 \mathrm{~b}$ ).

Na prática, a forma precisa das funções de velocidade de disparo $\left(\mathrm{F}_{\mathrm{e}}\right.$ e $F_{i}$ ) é determinada experimentalmente através da análise das velocidades de disparo dos neurónios do tálamo e do barril em resposta a um conjunto de deflecções bem seleccionadas (Pinto et al., 1996; Kyriazi, 1993). Quando as respostas simuladas estiverem de acordo com os dados experimentais, o modelo permitirá prever respostas a novos estímulos e compreender os mecanismos através dos quais aquelas respostas são geradas.

Uma previsão do modelo de actividade do barril é que a intensidade da resposta excitatória é sensível à distribuição dos sinais de entrada da população talâmica.

a)
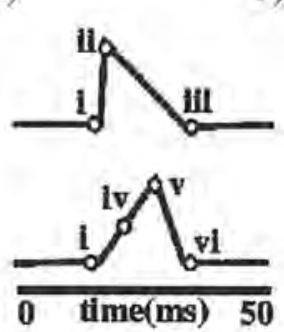

b)
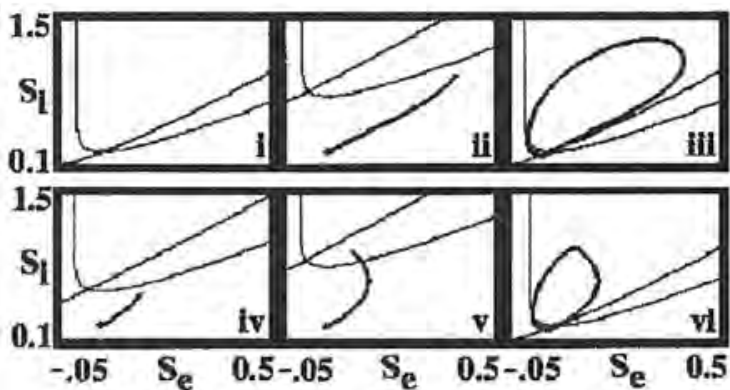

Fig. 5 - Análise do plano de fases de resposta simulada do barril a distribuições de entrada talâmicas. a) Duas distribuiçôes de entrada talâmicas, diferindo no curso temporal, mas com a mesma actividade total; b) Representação no plano de fases de respostas da população do barril. Planos sucessivos mostram as respostas progressivas nos instantes de tempo 
Para compreender este facto, examinaremos o plano de fases obtido a partir do modelo, em resposta a entradas de duas populações talâmicas (Pinto et al., 2001). No plano de fases, representa-se graficamente no eixo dos $x$ e no eixo dos $y$ o nível de actividade das populações excitatórias e o das inibitórias, respectivamente. Quando ocorre um sinal de entrada são traçados níveis de alteração da actividade pela trajectória de resposta nos sucessivos planos, nos instantes de tempo indicados pelos números romanos (figura 5 a e 5 b). A linha curva a fino (recta) é a linha de inclinação nula excitatória (inibitória), constituida por pontos nos quais $d S_{e} / d t=0\left(d S_{i} / d t=0\right)$.

Para o sinal de entrada talâmico de crescimento rápido, as linhas de inclinação nula sobem rapidamente (figura $5 \mathrm{~b}$, i a ii). Para o sinal de entrada de crescimento lento, as linhas de inclinação nula sobem mais lentamente. A trajectória-resposta permanece próxima da linha de inclinação nula onde as alterações na actividade são relativamente menores (figura $5 \mathrm{~b}$, i a iv a v). Assim, apesar de ambos os sinais de entrada representarem a mesma quantidade total de actividade talâmica (figura 5a), a distribuição de entrada de crescimento rápido gera uma resposta muito maior da rede de trajectórias do que a da distribuição de entrada de crescimento lento (figura $5 \mathrm{~b}$, iii versus vi). Esta previsão do modelo tem sido verificada experimentalmente através da análise da resposta de neurónios reais a diferentes distribuições de entrada talâmicas (Pinto et al., 2000).

\section{Das pinturas rupestres à estrutura do Córtex}

Durante anos as pessoas têm sentido um grande fascinio pelas pinturas rupestres do Paleolítico, tal como as que foram encontradas nas famosas cavernas de Lascaux, em França, e nas paredes de arenito no Sudoeste da América. Estas gravuras descrevem muitas vezes animais e seres humanos. Contudo, há também desenhos mais abstractos, nomeadamente espirais, raios solares e mandalas (círculos). Uma das questões que os antropólogos se colocam é saber qual é o significado destes símbolos abstractos. Um certo número de investigadores sugeriu que estas pinturas foram desenhadas por «xamãs» («homens-medicina») durante estados de transe e que os desenhos representam imagens visuais que resultam de um estado alterado de consciência (Hedges, 1981; Lewis-Williams, 1984; Hudson e Lee, 1984). Em particular, alguns antropólogos estabelecem uma analogia entre as formas icónicas da arte rupestre e as configurações descritas em fases iniciais de alucinação (Kluver, 1996), bem como as 
configurações que surgem em reacção a certos estímulos visuais, por exemplo, a pressão do globo ocular e a luz trémula (Tyler, 1978). Estas últimas configurações, chamadas fotópsias, e os seus análogos induzidos por estupefacientes foram classificadas por Kluver em quatro tipos diferentes, que designou "constantes de forma»:

- túneis e funis

- espirais

- reticulados, tais como favos de mel e tabuleiros de damas

- teias de aranha

Na figura 6 são apresentadas imagens representativas destas formas. Assim, de acordo com os antropólogos, os desenhos geométricos abstractos encontrados nas cavernas e nas pinturas em pedra são descrições de imagens vislumbradas por xamãs que estariam em estados mentais induzidos por transe ou por efeitos de «drogas». De facto, como iremos ver, a maioria dos desenhos mais comuns podem ser previstos através da análise da estrutura de configurações da actividade neuronal no córtex visual.

Vimos como se passa de modelos biofísicos pormenorizados para modelos reduzidos que permitem estudar circuitos sensoriais locais. Que acontece quando muitos desses circuitos são acoplados de um modo espacialmente estruturado? O córtex visual estriado é notável devido à sua organização, quase cristalina, de células que respondem à orientação, cor e posição no campo visual.

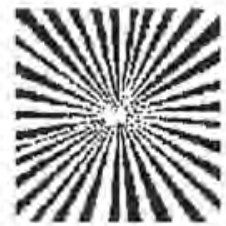

(1)

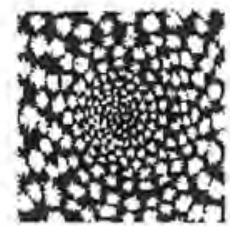

(II)

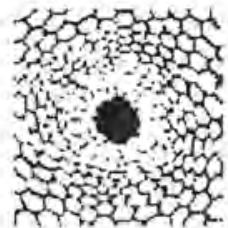

(III)

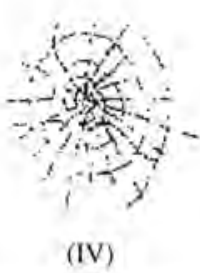

(IV)

Fig. 6-Constantes de forma (I) funil, (II) espiral, (III) reticulado, (IV) teia de aranha

Ou seja, o córtex visual está organizado em colunas (à semelhança dos barris) e no interior de cada coluna estão neurónios que respondem a (i) posições espaciais na retina; (ii) sinais de entrada dos olhos esquerdo e direito; (iii) linhas orientadas; outras modalidades visuais. As ligações 
entre as células não são aleatórias. Por exemplo, as células numa coluna que respondem a linhas verticais estão mais fortemente ligadas a células da mesma orientação em colunas vizinhas. As consequências desta ligação altamente estruturada têm sido objecto de análise num vasto número de artigos. Ermentrout e Cowan (1979) mostraram que as partições simétricas espontâneas em redes corticais espacialmente organizadas poderão justificar as configurações grosseiras da actividade espacial registadas durante alucinações. Mais recentemente, Bressloff et al. (2000) consideraram o acoplamento entre células sensiveis à orientação para explicar outros tipos de configurações alucinatórias mais complexas. No final desta secção, recorreremos a estas noções para compreender o contexto em que se poderão inserir os desenhos das cavernas acima descritos.

Comecemos por considerar uma arquitectura muito geral da rede neuronal descrita por:

$$
\tau_{i} \frac{d u_{i}}{d t}=-u_{i}+F_{i}\left(\sum_{j} W_{i j} u_{j}\right)
$$

que representa as actividades de muitos neurónios acoplados. $\mathrm{W}_{\mathrm{ij}}$ representa a intensidade das ligações entre neurónios, pelo que a equação (5) é uma generalização da equação (4). Esta rede pode ser separada em camadas. Suponhamos que eliminamos completamente a actividade de «fundo» («background») da rede, pelo que $\mathrm{u}_{\mathrm{i}}(\mathrm{t})$ representa o desvio do «fundo» e $\mathrm{F}_{\mathrm{i}}(0)=0$. Para simplificar pomos $\tau_{\mathrm{i}}=1$. Poderemos perguntar se este estado de "fundo" é ou não estável. Para esse efeito, precisamos de linearizar em torno do estado de repouso $u=0$. A estabilidade é determinada através dos valores próprios da matriz:

$$
M=-I+Q
$$

onde $Q_{i j}=F_{i}(0) W_{i j}$. Se Q for bastante pequeno (ou seja, há apenas uma fraca ligação entre as unidades ou $F_{i}^{\prime}(0)$ é pequeno), então os valores próprios de $\mathrm{M}$ têm partes reais negativas e todas as perturbações decaem para o estado de «fundo». Contudo, suponhamos que alguma influência externa (e.g., luz trémula, alucinogéneos, etc.) «excita» a rede tornando, por exemplo, $F_{i}$ elevadas. Então é possivel que alguns dos valores próprios de $\mathrm{M}$ venham positivos, o que conduz a instabilidades. A «forma» das actividades resultantes desta instabilidade é uma combinação de todos os vectores próprios de Q cujos valores próprios se tornaram simultanea- 
mente positivos. Genericamente, para uma rede simétrica aleatória, espera-se uma bifurcação «transcrítica» ou em «forquilha» conduzindo a uma forma excitada única que reflecte a organização aleatória da rede. Se o efeito dos alucinogéneos ou de outros estímulos externos é produzir um aumento geral de excitabilidade da rede, então é possivel que o estado de «fundo» possa tornar-se instável conduzindo a uma configuração espontânea de actividade que reflecte a organização associativa da rede recorrente. Se existem muitos valores próprios praticamente idênticos, então as configurações resultantes podem ser misturas complexas dos vectores próprios e das dinâmicas que regem as configurações.

Consideremos, por exemplo, uma matriz associativa de pesos,

$$
W_{i j}=\sum_{k=1}^{m \prime \prime} \xi_{i}^{k} \xi_{j}^{k}
$$

onde $\xi^{1}, \xi^{2}, \cdots, \xi^{\prime \prime \prime}$ são $m$ vectores a memorizar. Se cada um deles tem amplitude 1 e são quase ortogonais, então a matriz peso, W, tem um valor próprio $\lambda=1$ de multiplicidade $m$, sendo estes $m$ vectores os vectores próprios associados. Os métodos padrão de bífurcação podem ser aplicados para deduzir um conjunto de equações para os $m$ vectores próprios com a forma:

$$
A_{i}=v A_{i}+p_{2}\left(A_{1}, \ldots, A_{m}\right)+p_{3}\left(A_{1}, \ldots, A_{m}\right)
$$

onde $v$ é um parâmetro que controla a estabilidade do estado de «fundo» e $\mathrm{p}_{2}, \mathrm{p}_{3}$ são termos quadráticos e cúbicos, respectivamente. A configuração da actividade que surge passado o ponto de bifurcação tem a forma:

$$
\mathrm{u}(\mathrm{t}) \approx \mathrm{A}_{\mathrm{I}}(\mathrm{t}) \xi^{1}+\ldots+\mathrm{A}_{\mathrm{m}}(\mathrm{t}) \xi^{\mathrm{m}} .
$$

Portanto, para uma rede associativamente ligada, as configurações de actividade resultantes, que ocorrem quando o estado de «fundo» se torna instável, são misturas dinamicamente variadas de várias «memórias formadas».

Que tem isto a ver com «constantes de forma»? A análise acima esquematizada assume que a matriz-peso, $\mathrm{W}_{\mathrm{ij}}$, não tem estrutura ou tem uma estrutura proveniente de memórias formadas. Em áreas associativas do cérebro, é razoável considerar esta hipótese. Contudo, como mencionámos acima, o córtex vísual possui uma certa estrutura. As ligações estão longe de ser aleatórias e têm numerosas simetrias intrínsecas. Por exemplo, se a matriz dos pesos, W, é a associada a uma malha espacial bidimensional, com ligações que dependem apenas da distância, então os vectores próprios correspondentes a W têm uma estrutura periódica dupla 
(e,g., tiras, hexágonos ou pontos). As configurações resultantes que surgem na malha bidimensional são combinações de padrões formados por tiras e pontos.

Como poderá ser apreendida uma configuração de tiras no córtex? A resposta é a chave para a compreensão das constantes de forma de Kluver. Considere-se uma configuração de luz imposta na retina, Uma vez que há um «mapa» topográfico da retina para o córtex visual, isto resultará numa configuração no córtex. Reciprocảmente, cada configuração espacial de activação no córtex está associada a uma configuração virtual na retina. Em particular, Schwartz (1977) mostrou que cada ponto $(r, \theta)$ da retina, em coordenadas, está associado a um ponto $(x, y)$ no córtex. A transformação dada por:

$$
x=\frac{\alpha}{\varepsilon} \ln \left(1+\frac{\varepsilon}{\omega_{0}} r\right), \quad y=\frac{\beta r \cdot \theta}{\omega_{0}+\varepsilon r},
$$

e a inversa desta,

$$
r=\frac{\omega_{0}}{\varepsilon}\left(e^{\frac{x \varepsilon}{\alpha}}-1\right), \quad \theta=\frac{\varepsilon y}{\beta\left(1-e^{-\frac{x \varepsilon}{\alpha}}\right)},
$$

permite-nos transformar uma configuração cortical na sua análoga retinal. Perto do ponto central (fovea), a transformação é a identidade em escala. Longe desse ponto central (fovea), a transformação é o logaritmo complexo e a sua inversa é a exponencial complexa. A figura 7 mostra a acção desta transformação e o respectivo efeito numa constante de forma de tipo funil e de tipo espiral.

Esta transformação conjugada com a noção de que as alucinações visuais resultam da perda de estabilidade, primeiro no córtex estriado e posteriormente em áreas de associação mais elevada, permite-nos explicar as constantes de forma de Kluver e explicar indirectamente os desenhos abstractos das gravuras xamanistas. Ermentrout e Cowan (1979) estudaram a simetria de ligações com base na posição espacial e utilizaram a teoria de grupos para mostrar que as configurações espaciais que bifurcam a partir do estado de «fundo» não são mais que tiras e pontos. A forma normal das equações (6) possui uma estrutura muito simplificada. Por exemplo, surge a seguinte forma:

$$
A_{1}=v A_{1}-A_{1}\left(a A_{1}^{2}+b A_{2}^{2}\right)
$$




$$
\dot{A}_{2}=v A_{2}-A_{2}\left(a A_{2}^{2}+b A_{1}^{2}\right)
$$

onde $a, b$ são constantes dependentes do modelo e $A_{1}$ é a amplitude correspondente às tiras horizontais de actividade, enquanto $\mathrm{A}_{2}$ é a amplitude correspondente às tiras verticais. Se $A_{1} \neq 0, A_{2}=0\left(A_{1}=0\right.$, $A_{2} \neq 0$ ), então ocorrem tiras horizontais (verticais), enquanto se $\mathrm{A}_{1}=\mathrm{A}_{2}$, então ocorrem pontos. A estabilidade destas configurações de bifurcação depende apenas das constantes $a, b$. A figura 8 mostra exemplos de tiras horizontais e pontos de actividade em coordenadas corticais (em cima à esquerda), bem como a imagem observada em coordenadas retinais (em cima à direita).

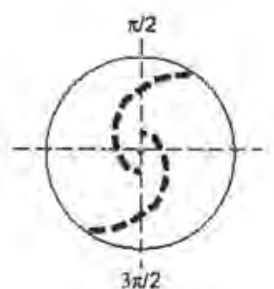

Visual Field
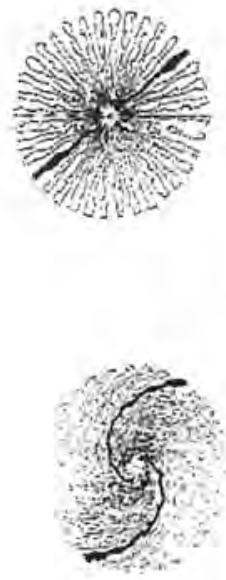

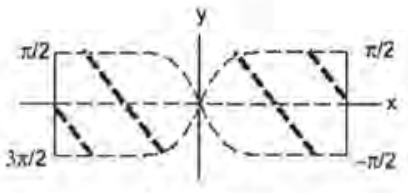

Striate Cortex

a)
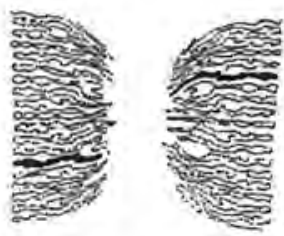

b)
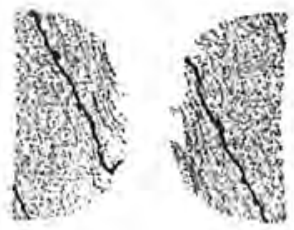

c)

Fig. 7 - (a) A transformação retinal $\rightarrow$ corlical (retina $\rightarrow$ córtex) e sua acção, a (b) a constante de forma "funily e (c) a constante de forma sespiral» 

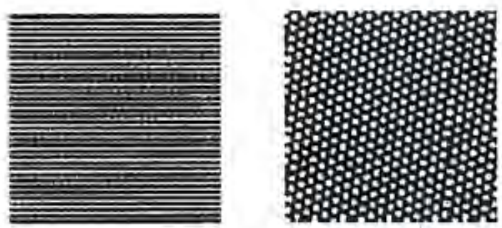

A
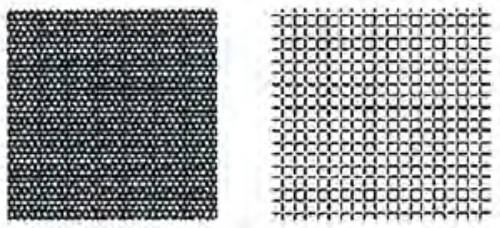
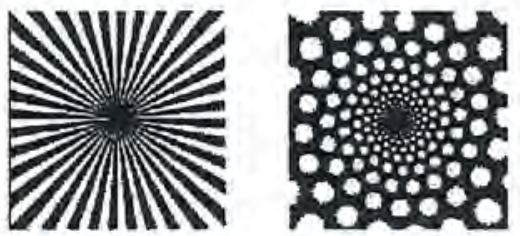

B

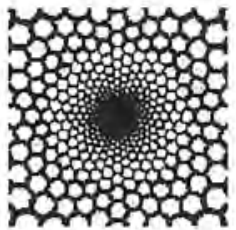

Fig. 8 - Configurações de bifurcação em coordenadas corticais (à esquerda) e em coordenadas retinais (à direita). As imagens superiores correspondem a configuraçôes originadas por ligações dependentes da distância e as inferiores correspondem a ligaçôes dependentes da distância e da orientaçăo (segundo Bressloff, et al.)

Se a estrutura da matriz de conexão incorpora a orientação específica de neurónios corticais, então os vectores próprios correspondentes são mais complicados, mas são interpretados pelo sistema visual como segmentos de recta dispostos em filas periódicas. Bressloff et al. (2000) analisaram este caso e deduziram as equações das formas normais. As configurações que correspondem aos pontos fixos destas equações são mais complexas do que simples tiras e pontos. Contudo, completam a enumeração das constantes de forma. As imagens inferiores da figura 8 mostram dois exemplos que correspondem a constantes de forma do tipo reticulado e do tipo teia de aranha.

\section{Referências}

Bressloff, P. C., Cowan, J. D., Golubitsky, M., Thomas, P. J., Wiener, M. (2000) Geometric visual hallucinations, Euclidean symmetry, and the functional architecture of striate cortex, Proc. Royal Society (a publicar).

Del Negro, C. A., Hsiao, C. F., Chandler, S. H., Garfinkel, S. (1998) - Evidence for a novel bursting mechanism in rodent trigeminal neurons. Biophys. J. 75, 174-182. Ermentrout, G. B, e Cowan, J. D., (1979) - A Mathematical Theory of Visual Hallucinations, Kybernetic 34: 137-150. 
Ermentrout, G. B., (1985) - A model for premigrainous auras. In The Neurobiology of Pain. ed. A. H. Holden e W. Winlow. Manchester University Press: Manchester.

Ermentrout, G. B., (1998) - Neuronal Nets as spatio-temporal pattern forming systems. Rep. Prog. Phys. 61: 353-430.

Gutkin, B. S, e Ermentrout, G. B., (2001) - How neurons multiplex coding of fast and slow stimuli (em preparação).

Gutkin, B. S,, e Ermentrout, G. B., (1998) - Dynamics of membrane excitability determine interspike interval variability: a link between spike generation mechanisms and cortical spike train statistics. Neuronal Comput. 10(5): $1047-1065$.

Hedges, K., (1981) - Phosphenes in the context of Native American rock art, in American Indian Rock Art vol VII, VIII, ed. FG Bock.

Hudson, T., e Lee, G. (1984) - Function and symbolism in Chumash rock art. J. New World Archaeology 6: 26-47.

Hodgkin, A. L., e Huxley, A. F. (1952) - A quantitative description of membrane current and its application to conduction and excitation in nerve. J. Phys. (Londres) 117: 500-544.

Jaeger, D., e Bower, J. M. (1999) - Synaptic control of spiking in cerebellar Purkinje cells: dynamic current clamp based on model conductances. $J$. Neurosci. 19(14): 6090-101.

Johnston, D., e Wu, S., (1997) - Foundations of cellular neurophysiology. MIT Press.

Kluver, H., (1996) - Mescal and the Mechanisms of Hallucination. University of Chicago Press: Chicago

Kyriazi, H. T., e Simons, D. J., (1993) - Thalamocortical response transformations in simulated whisker barrels. J. Neurosci. 13: 1601-1615.

Lancaster, B., e Adams, P. R., (1986) - Calcium-dependent current generating the after-hyperpolarization of hippocampal neurons. J. Neurophys. 55(6): 1268-82.

Le Masson, G,, Le Masson, S, Moulins, M., (1995) - From conductances to neuronal network properties: analysis of simple circuits using hybrid network method. Prog. Bioplys. Mol. Biol. 64(2-3): 201-20.

Lewis-Williams, J. D. e Dowson, T. A. (1988) - The signs of all times: entoptic phenomena in upper Paleolithic art. Current Antropology 29: 201-245.

Mainen, Z. F. e Sejnowski, T. S. (1995)-Reliability of spike timing in neocortical neurons. Science 268: 1503-1506.

McCormick, D. A., e Huguenard, J. R., (1992) - A Model of the Electrophysiological Properties of Thalamocortical Relay Neurons. J. Neurophys. $68: 1384-400$.

Pinto, D. J., Brumberg, J. C., Simons D. J., e Ermentrout, G. B., (1996) - A quantitative population model of whisker barrels: Re-examining the Wilson-Cowan equations. J. Comput. Neurosci. 3: 247-264. 
Pinto, D. J., Brumberg, J. C., Simons, D. J., (2000) - Circuit dynamics and coding strategies in rodent somatosensory cortex. J. Neurophysiol. 83: 1158-1166.

Pinto, D. J., Hartings, J. D., Brumberg, J. C., Ermentrout, G. B., Simons, D. J., (2001) - Recurrent excitation in damping cortical circuits: An alternative mechanism for response tuning. J. Neurosci (submetido).

Rall, W., e Agmon-Snir, A. (1998) - Cable theory for dendritic neurons, In Methods in Neuronal Modeling. C Koch, I Segev, eds. MIT Press Cambridge: MA. pp. 27-93.

Rinzel, J,, e Ermentrout, G. B., (1998) - Analysis of neuronal excitability and oscillations. In Methods in Neuronal Modeling. C Koch, I Segev, eds., MIT Press Cambridge: MA.

Scwartz, E., (1977) - Spatial mapping in the primary sensory projection: analytic structure and relevance to projection. Biol. Cybernefics 25: 181-194.

Shadlen, W. M., e Newsome, W. T., (1998) - The variable discharge of cortical neurons: implications for connectivity, computation and information coding. J. Neurosci. 18: 3870-96.

Sharp, A. A., O'Neil, M. B., Abbott, L. F., e Marder, E., (1993) - Dynamic clamp: computer-generated conductances in real neurons. J. Neurophysiol. 69 (3): 992-5.

Simons, D. J. (1978) - Response properties of vibrissa units in rat SI somatosensory neocortex. J. Neurophysiol. 41: 798-820.

Stafstrom, C. E., Schwindt, P. C., e Crill, W. E., (1982) - Negative slope conductance due to a persistent subthreshold sodium current in cat neocortical neurons in vitro. Brain Res. 236: 221-226

Tyler, C. (1978) - Some new entoptic phenomena. Vision Research 181: 1633-1639.

White, E. L, (1989) - Cortical Circuits: Synaptic organization of the cerebral cortex. Birkhauser: Boston, MA.

Woolsey, T. A, van der Loos, H., (1970) - The structural organization of layer IV in the somatosensory region (SI) of mouse cerebral cortex. Brain Res. 17: $205-242$. 


\section{$\begin{array}{llllll}\text { C I E } & \mathbf{N} & \mathbf{C} & \mathbf{A}\end{array}$}

mitiram realizar com sucesso tarefas tão distintas como a programação de um voo a Marte, a previsão de resultados eleitorais, a explicação do funcionamento de alguns mecanismos do sistema nervoso, ou a abordagem critica de obras de arte e de textos literários. Da ciência à sociedade, dos grandes avanços técnicos à solidez de uma argumentação lógica, a Matemática constrói teias de uma imensa flexibilidade resultante do carácter universal da sua linguagem.

Neste livro, personalidades de diferentes universos dão o seu testemunho sobre a forma como usam as teias matemáticas para tecer a sua própria visão do mundo.

Maria Paula Serra de Oliveira é professora de Matemática na Faculdade de Ciências e Tecnologia da Universidade de Coimbra. 